\title{
Kochen-Specker Theorem for a Single Qubit Using Positive Operator-Valued Measures
}

\author{
Adán Cabelld* \\ Departamento de Fúsica Aplicada II, Universidad de Sevilla, 41012 Sevilla, Spain
}

(Dated: October 26, 2018)

\begin{abstract}
A proof of the Kochen-Specker theorem for a single two-level system is presented. It employs five eight-element positive operator-valued measures and a simple algebraic reasoning based on the geometry of the dodecahedron.

PACS numbers: 03.65.Ta, 03.65.Ud, 03.67.-a
\end{abstract}

It is a widely held belief that "a single qubit is not a truly quantum system in the sense that its dynamics and its response to measurements can all be mocked up by a classical hidden-variable model. There are no Bell inequalities or a Kochen-Specker theorem for a twodimensional system that forbids the existence of a classical model" [1]. Any proof of Bell's theorem of the impossibility of local hidden-variables in quantum mechanics requires a composite system. On the other hand, the standard proof of the Kochen-Specker (KS) theorem [2, 3, 4] of the impossibility of noncontextual hiddenvariables applies only to physical systems described by Hilbert spaces of dimension three or higher.

In this Letter, I show that it is possible to extend the KS theorem to a single two-level system (qubit). The key is to consider generalized measurements, represented by positive operator-valued measures (POVMs) [5, 6, 7, 8, [9], instead of just standard measurements, represented by von Neumann's projection-valued measures. This shall lead to a generalization of the KS theorem which rules out all hidden-variable theories ruled out by the original KS theorem plus some hidden-variable theories for a single qubit. The common feature of all these hidden-variable theories is that they are noncontextual, that is, they assign predefined yes-no answers to a set of questions $\{Q, R, S \ldots\}$ (tests) independently of whether question $Q$ is formulated jointly with question $R$ or with a different question $S$. The physical content of the KS theorem can also be summarized by saying that "measurements" do not reveal preexisting values, or that "unperformed experiments have no results" [10].

The standard proof of the KS theorem is based on the observation that, for a physical system described by a Hilbert space of dimension $d \geq 3$, it is possible to find a set of $n$ projection operators, which represent yes-no questions about the physical system, so that none of the $2^{n}$ possible sets of "yes" or "no" answers is compatible with the sum rule for orthogonal resolutions of the identity (i.e., if the sum of a subset of mutually orthogonal projection operators is the identity, one and only one of the corresponding answers ought to be yes) [8, 11]. The original proof required 117 projection operators in $d=3[4]$. The actual record stands at 18 projection operators in $d=4$ [12]. The record for $d=3$ stands at 31 projection operators 13 .
It is impossible to prove the KS theorem for a single qubit (described by a Hilbert space of $d=2$ ) by using a set of projection operators. Any proof of this kind would require that any projection operator of the set be orthogonal to at least two other projection operators. However, in $d=2$ any projection operator is orthogonal only to one projection operator. Therefore, for $d=2$, it is possible to assign yes and no answers to all projection operators, satisfying the sum rule for orthogonal resolutions of the identity. This explains why it is possible to construct explicit noncontextual hidden-variable models that are capable of reproducing all the predictions of quantum mechanics for von Neumman's measurements on a single qubit 3, 4, 14, 15, 16.

Motivated by the quantum information approach to quantum mechanics and by the fact that current technology allows an exquisite level of control over the measurements that can be performed, recent formulations of the principles of quantum mechanics [8, 9, 17] stress that the measurements correspond to POVMs, extending the notion of von Neumann's projection-valued measures. The main difference between POVMs and von Neumann's projection-valued measures is that for POVMs the number of available outcomes of a measurement may be higher than the dimensionality of the Hilbert space. An $N$-outcome generalized measurement is represented by an $N$-element POVM which consists of $N$ positive-semidefinite operators $\left\{E_{d}\right\}$ that sum the identity (i.e., $\sum_{d} E_{d}=\mathrm{I}$ ). Neumark's theorem [5] guarantees that there always exists a realizable experimental procedure to generate any desired POVM. Any generalized measurement represented by a POVM can be seen as a von Neumann's measurement on a larger Hilbert space. Therefore, any generalized measurement on a single qubit can be seen as a von Neumann's joint measurement on a system composed by the qubit plus an auxiliary quantum system (ancilla) [18]. If we define the ancilla as belonging to the measuring apparatus, then we can legitimately speak of a (generalized) measurement on a single qubit [19].

It has been shown that, when one considers an ancilla, then noncontextual hidden-variables cannot reproduce the predictions of quantum mechanics for von Neumman's measurements on pre- and post-selected systems [20]. On the other hand, the KS theorem can be 
seen as a consequence of Gleason's theorem [21]. Recently, a Gleason-like theorem using POVMs has been proved 22, 23]. Unlike Gleason's theorem, the new one is also valid for $d=2$. This suggests that the KS theorem could be extended to $d=2$ by using POVMs instead of von Neumann's measurements [17].

Physically, this would mean that it is impossible to construct a noncontextual hidden-variables theory for a single qubit which assigns an outcome, for instance $\mathcal{E}_{A}$, regardless of whether this outcome belongs to a the POVM represented by $\mathcal{E}_{A}, \mathcal{E}_{B}, \ldots, \mathcal{E}_{M}$ or to the POVM represented by $\mathcal{E}_{A}, \mathcal{E}_{b}, \ldots, \mathcal{E}_{m}$. This bears a close similarity to the original formulation of the KS theorem [4] based on von Neumann's measurements on a single spin-1 system. KS considered measurements of the type

$$
H(x, y, z)=a S_{x}^{2}+b S_{y}^{2}+c S_{z}^{2},
$$

where $a, b$, and $c$ are real distinct numbers and $S_{x}^{2}$ is the square of the spin component along the $x$ direction. A measurement of $H$ has three possible outcomes: $b+c, a+c$, and $a+b$. KS showed that whichever outcome actually occurs was not predefined. They accomplished this by considering alternative measurements $H(x, j, k)$, where $x, j$, and $k$ are mutually orthogonal directions, and assuming that if the outcome of measuring $H(x, y, z)$ had/had not been $b+c$, then the outcome of measuring $H(x, j, k)$ would have/have not been $b+c$.

The challenge is to prove the KS theorem for $d=2$ using generalized measurements, that is, to find an explicit set of POVMs on a single qubit so that none of the possible sets of $2^{n}$ yes or no answers (where $n$ is the number of different positive-semidefinite operators in the POVMs) is compatible with the sum rule for positivesemidefinite operators of a POVM (i.e., if the sum of a subset of positive-semidefinite operators is the identity, one and only one of the corresponding answers ought to be yes). Fuchs has suggested using sets of three-outcome POVMs of the "Mercedes-Benz" type 17]. So far, however, no proof with this or any other type of POVMs has been described.

Let us define the following eight-outcome generalized measurement on a qubit represented by the following eight-element POVM: $\left\{\mathcal{E}_{C+}, \mathcal{E}_{C_{-}}, \mathcal{E}_{E+}, \mathcal{E}_{E-}, \mathcal{E}_{F+}, \mathcal{E}_{F-}, \mathcal{E}_{G+}, \mathcal{E}_{G-}\right\}$, where

$$
\begin{aligned}
\mathcal{E}_{C+} & =\frac{1}{4} P_{\neg|C=-1\rangle}=\frac{1}{4}\left(\mathbb{I}-P_{|C=-1\rangle}\right) \\
& =\frac{1}{4}|C=+1\rangle\langle C=+1|, \\
\mathcal{E}_{C-} & =\frac{1}{4} P_{\neg|C=+1\rangle}=\frac{1}{4}\left(\mathbb{I}-P_{|C=+1\rangle}\right) \\
& =\frac{1}{4}|C=-1\rangle\langle C=-1|,
\end{aligned}
$$

and analogously $\mathcal{E}_{E+}$, etc. $C, E, F$, and $G$ are the directions obtained by connecting the center of a cube with

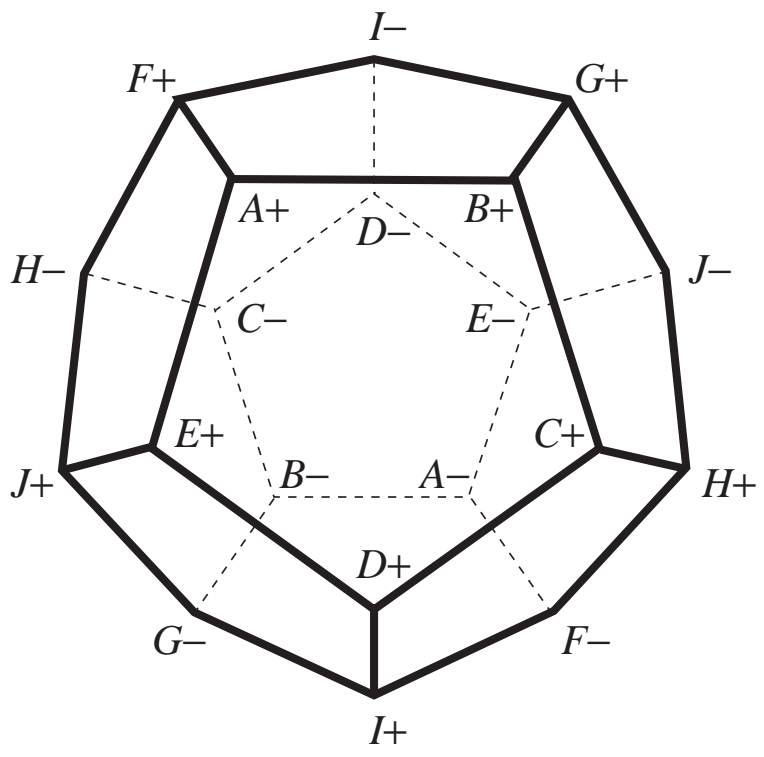

FIG. 1: Notation for the 20 vertices of the dodecahedron: $A+$ is the antipode of $A-$.

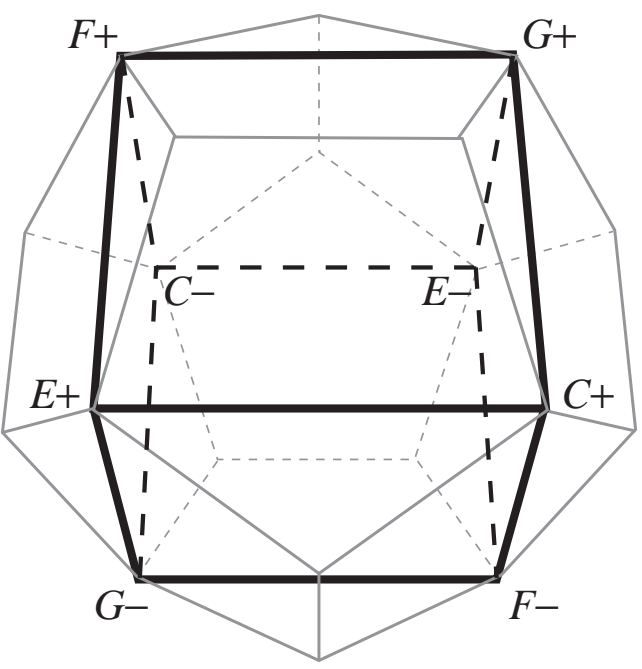

FIG. 2: The cube $C E F G$ is one of the five inscribed (sharing vertices) in the dodecahedron. It corresponds to an eightelement POVM.

its four nonantipodal vertices. $P_{\neg|C=-1\rangle}$ is the projection on the qubit states orthogonal to $|C=-1\rangle$ (which, for example, could be the spin state along direction $C$ with eigenvalue -1 of a spin- $1 / 2$ particle). As can be easily checked, the sum of these eight positive-semidefinite operators is the identity.

Now let us consider the ten directions obtained by connecting the center of a dodecahedron with its ten nonantipodal vertices, labelled $A, B, \ldots, J$ as in Fig. 1 There are only five cubes inscribed (sharing vertices) in a dodecahedron (Fig. 2). All of them share the same center, and any two cubes share two antipodal vertices. Each cube allows us to define an eight-element POVM similar 
to the one defined above. The resulting five POVMs can be expressed as:

$$
\begin{gathered}
\mathcal{E}_{A+}+\mathcal{E}_{A-}+\mathcal{E}_{C+}+\mathcal{E}_{C-}+ \\
\mathcal{E}_{I+}+\mathcal{E}_{I-}+\mathcal{E}_{J+}+\mathcal{E}_{J-}=\mathbb{I} \\
\mathcal{E}_{A+}+\mathcal{E}_{A-}+\mathcal{E}_{D+}+\mathcal{E}_{D-}+ \\
\mathcal{E}_{G+}+\mathcal{E}_{G-}+\mathcal{E}_{H+}+\mathcal{E}_{H-}=\mathbb{I}, \\
\mathcal{E}_{B+}+\mathcal{E}_{B-}+\mathcal{E}_{D+}+\mathcal{E}_{D-}+ \\
\mathcal{E}_{F+}+\mathcal{E}_{F-}+\mathcal{E}_{J+}+\mathcal{E}_{J-}=\mathbb{I}, \\
\mathcal{E}_{B+}+\mathcal{E}_{B-}+\mathcal{E}_{E+}+\mathcal{E}_{E-} \\
\mathcal{E}_{H+}+\mathcal{E}_{H-}+\mathcal{E}_{I+}+\mathcal{E}_{I-}=\mathbb{I}, \\
\mathcal{E}_{C+}+\mathcal{E}_{C-}+\mathcal{E}_{E+}+\mathcal{E}_{E-}+ \\
\mathcal{E}_{F+}+\mathcal{E}_{F-}+\mathcal{E}_{G+}+\mathcal{E}_{G-}=\mathbb{I} .
\end{gathered}
$$

Each equation contains eight positive-semidefinite operators whose sum is the identity. Therefore, a noncontextual hidden-variable theory must assign the answer yes to one and only one of these eight operators. However, such an assignment is impossible, since each operator appears twice in (41)-(8), so that the total number of yes answers must be an even number, while the number of POVMs, and thus the number of possible yes answers, is five.

Geometrically, this proof expresses the impossibility of coloring black (for yes) or white (for no) the vertices of a dodecahedron in such a way that each of the five inscribed cubes has one and only one vertex colored black.

To my knowledge, this is the first proof of the KS theorem for a single qubit. Moreover, this proof joins the three most wanted features in a proof of the KS theorem: (a) It is based on a simple algebraic argument (parity, like the proofs in 12, 24]), so checking the impossibility of coloring requires neither an intricate geometrical argument [4] nor a computer program [8]; (b) it needs few operators (five POVMs containing only 20 different positive-semidefinite operators); (c) it admits an elegant geometrical interpretation. Curiously indeed, this interpretation is in terms of the dodecahedron, whose geometrical properties were also used in Penrose's proofs of the KS theorem [25, 26, 27].

I never met Rob Clifton personally, but for years we maintained some correspondence on the KS theorem. He once told me: "Kochen-Specker problems can be addictive and I am an addict!" [28]. I share this addiction. I would like to dedicate this Letter to his memory. I would like to thank J. Bub, C. A. Fuchs, and A. Peres for comments, and the Spanish Ministerio de Ciencia y Tecnología Grants No. BFM2001-3943 and No. BFM200202815, and the Junta de Andalucía Grant No. FQM-239 for support.

Note added.-After reading an earlier version of this Letter, Masahiro Nakamura has found a simpler proof of the KS theorem for a single qubit: Let $A, B$, and $C$ be the three directions obtained by joining the center of a regular hexagon with its three nonantipodal vertices. A simple parity argument shows that it is impossible to assign noncontextual yes-no answers to the six positive semidefinite operators contained in the three four-element POVMs $\left\{\mathcal{E}_{A+}, \mathcal{E}_{A-}, \mathcal{E}_{B+}, \mathcal{E}_{B-}\right\},\left\{\mathcal{E}_{B+}, \mathcal{E}_{B-}, \mathcal{E}_{C+}, \mathcal{E}_{C-}\right\}$, and $\left\{\mathcal{E}_{A+}, \mathcal{E}_{A-}, \mathcal{E}_{C+}, \mathcal{E}_{C_{-}}\right\}, \quad$ where $\mathcal{E}_{A+}=\frac{1}{2}|A=+1\rangle\langle A=+1|$, etc. [29].

Electronic address: adan@us.es

[1] S.J. van Enk, Phys. Rev. Lett. 84, 789 (2000).

[2] E.P. Specker, Dialectica 14, 239 (1960).

[3] J.S. Bell, Rev. Mod. Phys. 38, 447 (1966).

[4] S. Kochen and E.P. Specker, J. Math. Mech. 17, 59 (1967).

[5] M.A. Neumark, Dokl. Akad. Nauk SSSR 41, 359 (1943).

[6] G. Ludwig, Einführung in die Grundlagen der Theoretischen Physik (Vieweg, Braunschweig, 1976).

[7] E.B. Davies, Quantum Theory of Open Systems (Academic Press, New York, 1976).

[8] A. Peres, Quantum Theory: Concepts and Methods (Kluwer Academic, Dordrecht, 1993).

[9] M.A. Nielsen and I.L. Chuang, Quantum Computation and Quantum Information (Cambridge University Press, Cambridge, 2000).

[10] A. Peres, Am. J. Phys. 46, 745 (1978). Reprinted in Foundations of Quantum Mechanics since the Bell Inequalities, Selected Reprints, edited by L.E. Ballentine (American Asocciation of Physics Techers, College Park, MD, 1988), p. 100.

[11] J. Bub, Interpreting the Quantum World (Cambridge University Press, Cambridge, 1997).

[12] A. Cabello, J.M. Estebaranz, and G. García Alcaine, Phys. Lett. A 212, 183 (1996).

[13] J.H. Conway and S. Kochen, first reported in [8], p. 114. See also J.H. Conway and S. Kochen, in Quantum [Un]speakables: From Bell to Quantum Information, edited by R.A. Bertlmann and A. Zeilinger (SpringerVerlag, Berlin, 2002), p. 257.

[14] D. Bohm and J. Bub, Rev. Mod. Phys. 38, 453 (1966).

[15] J.F. Clauser, Am. J. Phys. 39, 1095 (1971).

[16] F. Selleri, Quantum Paradoxes and Physical Reality (Kluwer Academic, Dordrecht, 1990), p. 48.

[17] C.A. Fuchs, quant-ph/0205039

[18] Since a generalized measurement represented by POVMs can only be implemented by means of a von Neumann's measurement on a higher dimensional system, and since the standard KS theorem shows that there cannot be a noncontextual hidden-variables theory for the higher dimensional system, then one might think that the impossibility of a noncontextual hidden-variables theory for the lower dimensional system follows from the impossibility of a noncontextual hidden-variables theory for the higher dimensional system. However, there is a need for a proof using POVMs similar to the one introduced in this Letter, because at present it has not been proved that a standard proof of the KS theorem in the higher dimensional system would lead to a proof of the KS theorem using POVMs in the lower dimensional system.

[19] According to Peres, "measurements are processes in which an apparatus interacts with the physical system under study, in such a way that a property of that system affects a corresponding property of the apparatus" [8]. 
The approach to the KS theorem using POVMs is fully consistent with this definition: here the physical system under study is a single qubit and the apparatus includes the ancilla.

[20] A. Cabello, Phys. Rev. A 55, 4109 (1997).

[21] A.M. Gleason, J. Math. Mech. 6, 885 (1957).

[22] P. Busch, quant-ph/9909073

[23] C.M. Caves, C.A. Fuchs, K. Manne, and J. Renes (unpublished, 2000).

[24] M. Kernaghan, J. Phys. A 27, L829 (1994).

[25] R. Penrose, in Quantum Reflections, edited by J. Ellis and D. Amati (Cambridge University Press, Cambridge, 2000), p. 1.

[26] J.R. Zimba and R. Penrose, Stud. Hist. Philos. Sci. 24, 697 (1993).

[27] R. Penrose, Shadows of the Mind: A Search for the Missing Science of Consciousness (Oxford University Press, Oxford, 1994), Chap. 5.

[28] R.K. Clifton (private communication, 1996).

[29] M. Nakamura (private communication, 2003). 